\title{
SOME ITERATED LOGARITHM RESULTS RELATED TO THE CENTRAL LIMIT THEOREM( $\left.{ }^{1}\right)$
}

\author{
BY \\ R. J. TOMKINS $\left({ }^{2}\right)$
}

\begin{abstract}
An iterated logarithm theorem is presented for sequences of independent, not necessarily bounded, random variables, the distribution of whose partial sums is related to the standard normal distribution in a particular manner. It is shown that if a sequence of independent random variables satisfies the Central Limit Theorem with a sufficiently rapid rate of convergence, then the law of the iterated logarithm holds. In particular, it is demonstrated that these results imply several known iterated logarithm results, including Kolmogorov's celebrated theorem.
\end{abstract}

1. Introduction. Since the appearance in 1929 of Kolmogorov's Law of the Iterated Logarithm [8] for a class of independent, bounded random variables (r.v.), a number of probabilists have attempted to determine conditions under which similar results would hold for unbounded random variables; some of these results will be discussed in $\$ 3$.

Consider a probability space $(\Omega, \mathscr{F}, P)$. In this paper, a new result of the iterated logarithm type, which requires no bounds on the sequence of random variables, will be presented. More specifically, it is the intention of this article to prove, and indicate some consequences of, the following theorem.

THEOREM 1. Let $X_{1}, X_{2}, \ldots$ be independent r.v. with $E X_{n}=0$ and $E X_{n}^{2} \equiv \sigma_{n}^{2}<\infty$.

Define $S_{n}=X_{1}+\cdots+X_{n}, s_{n}^{2}=E S_{n}^{2}, t_{n}^{2}=2 \log \log \left(s_{n}^{2}\right)$ and $F_{n}(x)=P\left[S_{n} \leqq x \cdot s_{n}\right]$, $-\infty<x<\infty$. Let $\Phi$ represent the standard normal distribution function. Suppose a sequence of real-valued functions $f_{n}$ are defined on an interval $(A, B)$ which contains 1 such that (i) $\lim f_{n}(x)=0$ for all $x \in(A, B)$, and (ii) for all $x \in(A, B)$,

$$
\limsup _{n \rightarrow \infty} \frac{1-F_{n}\left(x t_{n}\right)}{1-\Phi\left(x t_{n}\right)} \cdot \exp \left\{(1 / 2) x^{2} t_{n}^{2} f_{n}(x)\right\}
$$

is finite.

Received by the editors April 27, 1970.

AMS 1969 subject classifications. Primary 6030.

Key words and phrases. Independent random variables, law of the iterated logarithm, Central Limit Theorem, normal distribution, Berry-Esseen bounds, Lindeberg's criterion, Kolmogorov's theorem.

(1) The main result of the paper is a revised version of a theorem in the author's Ph.D. dissertation, prepared for Purdue University under the supervision of Dr. Y. S. Chow. The dissertation research was supported by National Science Foundation grant GPO7631.

${ }^{(2)}$ The author is indebted to the Principal's Fund of the University of Saskatchewan, Regina Campus, for research funds received during the preparation of this paper.

Copyright (C) 1971, American Mathematical Society 
If (iii) $s_{n} \rightarrow \infty$ and (iv) $\sigma_{n} / s_{n} \rightarrow 0$, then

$$
\limsup _{n \rightarrow \infty} S_{n} /\left(s_{n} t_{n}\right) \leqq 1 \quad \text { a.e. }
$$

If, furthermore, there exists a sequence $h_{n}$ of real-valued functions defined on $(A, B)$ such that $(\mathrm{v}) \lim h_{n}(x)=0$ for all $x \in(A, B)$, and (vi) for each $x \in(A, B)$,

$$
\liminf _{n \rightarrow \infty} \frac{1-F_{n}\left(x t_{n}\right)}{1-\Phi\left(x t_{n}\right)} \cdot \exp \left\{(1 / 2) x^{2} t_{n}^{2} h_{n}(x)\right\}
$$

is positive, and if, in addition, condition (ii) holds with $1-F_{n}\left(x t_{n}\right)$ replaced by $F_{n}\left(-x t_{n}\right)$, then

$$
\limsup _{n \rightarrow \infty} S_{n} /\left(s_{n} t_{n}\right)=1 \quad \text { a.e. }
$$

The proof of this theorem will be given in $\$ 2$. $\$ 3$ will contain some corollaries and consequences of Theorem 1 . In particular, the relationship between Theorem 1 and some of the previously published iterated logarithm results for unbounded random variables will be discussed.

By appealing to a sophisticated result of Feller [3], it will be shown in $\$ 4$ that Kolmogorov's Law of the Iterated Logarithm can be derived from Theorem 1.

Before beginning $\$ 2$, some remarks about notation are in order. Throughout the paper, $\Phi$ will denote the distribution function of the normal distribution with mean zero and variance one. Only natural logarithms will be used. For sequences $\left\{a_{n}\right\}$ and $\left\{b_{n}\right\}$ of positive real numbers, " $a_{n} \sim b_{n}$ " means that $\lim a_{n} / b_{n}=1$. And, finally, the well-known "limit comparison test" will be used repeatedly; to wit, if $a_{n} \sim b_{n}$, then $\sum_{n=1}^{\infty} a_{n}$ and $\sum_{n=1}^{\infty} b_{n}$ converge or diverge together.

2. Proof of Theorem 1. Before proceeding with the proof of the theorem, the following useful lemma will be proved.

Lemma 1. Let $\varepsilon>1$ be arbitrary. Then (i) $1-\Phi(\varepsilon)<\exp \left\{-\varepsilon^{2} / 2\right\}$ and (ii) for any given $\gamma>0$ there exists a number $\varepsilon_{0}>0$ (which depends on $\gamma$ ) such that, for all $\varepsilon \geqq \varepsilon_{0}, 1-\Phi(\varepsilon)>\exp \left\{-(1+\gamma) \varepsilon^{2} / 2\right\}$.

Proof. The result is immediate from the following well-known inequality (see p. 166 of [4], for example):

$$
(2 \pi)^{-1 / 2}\left(\varepsilon^{-1}-\varepsilon^{-3}\right) \exp \left\{-\varepsilon^{2} / 2\right\}<1-\Phi(\varepsilon)<(2 \pi)^{-1 / 2} \varepsilon^{-1} \exp \left\{-\varepsilon^{2} / 2\right\} .
$$

(i) is obvious, whereas (ii) follows if $\varepsilon$ is so large that

$$
\left(\varepsilon^{-1}-\varepsilon^{-3}\right)(2 \pi)^{-1 / 2} \exp \left\{\gamma \varepsilon^{2} / 2\right\}>1 . \quad \text { Q.E.D. }
$$

We are now in a position to prove Theorem 1. It is to be noticed that the proof owes a great debt to Kolmogorov.

Note, first of all, that it follows from (iv) that $s_{n} \sim s_{n+1}$ as $n \rightarrow \infty$.

Let $1<\alpha^{\prime \prime}<\alpha^{\prime}<\alpha<B$ be arbitrarily chosen and select a number $c>1$ so close to 1 that $\alpha>c \alpha^{\prime}$. 
Now, for each $k \geqq 1$, let $n_{k}$ be the least integer satisfying $s_{n_{k}}>c^{k}$; this sequence is well defined by virtue of (iii). It should be mentioned that $s_{n_{k}} \sim c^{k}$ and $t_{n_{k}} \sim t_{n_{k+1}}$ as $k \rightarrow \infty$. Furthermore, an integer $K_{0}>0$ exists such that

$$
n_{k-1}<n_{k} \text { and } 2^{1 / 2}<\left(\alpha^{\prime}-\alpha^{\prime \prime}\right) t_{n_{k}} \quad \text { for all } k \geqq K_{0}:
$$

For each $k \geqq K_{0}$, define $I_{k}=\left\{n \mid n_{k-1}<n \leqq n_{k}\right\}$, and $S_{k}^{*}=\max _{n \in I_{k}} S_{n}$. By a variation of Levy's inequality (see p. 248 of [9]), it follows from (3) that

$$
\begin{aligned}
P\left[S_{k}^{*} \geqq \alpha^{\prime} s_{n_{k}} t_{n_{k}}\right] & \leqq 2 P\left[S_{n_{k}} \geqq \alpha^{\prime} s_{n_{k}} t_{n_{k}}-2^{1 / 2} s_{n_{k}}\right] \\
& \leqq 2 P\left[S_{n_{k}} \geqq \alpha^{\prime \prime} s_{n_{k}} t_{n_{k}}\right]=2\left\{1-F_{n_{k}}\left(\alpha^{\prime \prime} t_{n_{k}}\right)\right\} \equiv a_{k} .
\end{aligned}
$$

By (ii), we may assume the existence of a real-valued function $U$ defined on $(A, B)$ such that

$$
\left[1-\Phi\left(x t_{n}\right)\right]^{-1}\left[1-F_{n}\left(x t_{n}\right)\right] \cdot \exp \left\{(1 / 2) x^{2} t_{n}^{2} f_{n}(x)\right\} \leqq U(x)
$$

if $n$ is sufficiently large. Furthermore, by (i), there exists $N>0$ such that $\alpha^{\prime \prime}\left(1+f_{n}\left(\alpha^{\prime \prime}\right)\right)>1$ for all $n>N$. Therefore, by Lemma 1(i), it is clear that for all sufficiently large $k$

$$
a_{k} \leqq 2 U\left(\alpha^{\prime \prime}\right) \exp \left\{-(1 / 2)\left(\alpha^{\prime \prime}\right)^{2} t_{n_{k}}^{2}\left(1+f_{n_{k}}\left(\alpha^{\prime \prime}\right)\right)\right\} \leqq 2 U\left(\alpha^{\prime \prime}\right)\left(\log s_{n_{k}}^{2}\right)^{-\alpha^{\prime \prime}} .
$$

But $\left(\log s_{n_{k}}^{2}\right)^{-\alpha^{\prime \prime}} \sim(2 \log c)^{-\alpha^{\prime \prime}} \cdot k^{-\alpha^{\prime \prime}}$, so $\sum_{k \geqq K_{0}} a_{k}<\infty$ since $\alpha^{\prime \prime}>1$. Hence

$$
\sum_{k \geqq K_{0}} P\left[S_{k}^{*}>\alpha^{\prime} S_{n_{k}} t_{n_{k}}\right]<\infty
$$

by (4); the Borel-Cantelli lemma then implies that

$$
P\left[S_{k}^{*} \geqq \alpha^{\prime} s_{n_{k}} t_{n_{k}} \text { i.o. }\right]=0 .
$$

But $s_{n_{k-1}} t_{n_{k-1}} \sim s_{n_{k}} t_{n_{k}} / c$ and $\alpha>c \alpha^{\prime}$, so that

$$
0 \leqq P\left[S_{n} \geqq \alpha S_{n} t_{n} \text { i.o. }\right] \leqq P\left[S_{k}^{*} \geqq \alpha S_{n_{k-1}} t_{n_{k-1}} \text { i.o. }\right] \leqq P\left[S_{k}^{*} \geqq \alpha^{\prime} s_{n_{k}} t_{n_{k}} \text { i.o. }\right]=0 .
$$

Obviously, then, lim $\sup _{n \rightarrow \infty} S_{n} /\left(s_{n} t_{n}\right) \leqq \alpha$ a.e.; (1) follows since $\alpha>1$ is arbitrary.

The second part of Theorem 1 will now be proved. Let $0<\varepsilon<\varepsilon^{\prime}<1-A$ be arbitrary. Let $b=(1+B) / 2$ and choose a number $c>1$ so large that

$$
\varepsilon\left(c^{2}-1\right)^{1 / 2}>3 b \text { and }(1-\varepsilon)\left(c^{2}-1\right)^{1 / 2}>1+c\left(1-\varepsilon^{\prime}\right) \text {. }
$$

For each $k \geqq 1$, define $n_{k}$ to be the least integer satisfying $s_{n_{k}}>c^{k}$. There exists a number $K_{1}>0$ such that $n_{k-1}<n_{k}$ for $k \geqq K_{1}$.

For each $k \geqq K_{1}$, let $u_{k}^{2}=s_{n_{k}}^{2}-s_{n_{k-1}}^{2}$, and $v_{k}^{2}=2 \log \log \left(u_{k}^{2}\right)$. Note that $u_{k}^{2}$ $\sim c^{-2}\left(c^{2}-1\right) s_{n_{k}}^{2} \sim\left(c^{2}-1\right) s_{n_{k-1}}^{2}$ and $t_{n_{k}} \sim v_{k}$ as $k \rightarrow \infty$, from which facts it is easily seen that $u_{k}^{2} v_{k}^{2} /\left(s_{n_{k-1}}^{2} t_{n_{k-1}}^{2}\right)>4\left(c^{2}-1\right) / 9$ for all large $k$.

In addition, define the following events for each $k \geqq K_{1}$ :

$$
A_{k}=\left[S_{n_{k}}-S_{n_{k-1}}>(1-\varepsilon) u_{k} v_{k}\right], \quad B_{k}=\left[S_{n_{k}}>(1-\varepsilon / 2) u_{k} v_{k}\right]
$$


and

$$
C_{k}=\left[S_{n_{k-1}}>\varepsilon u_{k} v_{k} / 2\right] .
$$

By a remark above and (5), $P C_{k} \leqq 1-F_{n_{k-1}}\left(b t_{n_{k-1}}\right)$. But $b\left(1+f_{n_{k-1}}(b)\right)>1$ for all large $k$. Hence, proceeding in a fashion analogous to a portion of the proof of the first part of the theorem, we can use (ii) and Lemma 1(i) to show that, for all sufficiently large $k$,

$$
P C_{k} \leqq U(b) \exp \left\{-(1 / 2) b t_{n_{k-1}}^{2}\right\} \sim U(b)(2 \log c)^{-b}(k-1)^{-b} .
$$

So $\sum_{k \geqq K_{1}} P C_{k}<\infty$ since $b>1$.

Now define $\beta=1-\varepsilon / 2$ and $\gamma=\beta^{-2}-1$. Then, since $u_{k} v_{k}<s_{n_{k}} t_{n_{k}}, P B_{k}>1-F_{n_{k}}\left(\beta t_{n_{k}}\right)$. By (vi), a positive function $L$ is defined on $(A, B)$ such that

$$
\left[1-\Phi\left(x t_{n}\right)\right]^{-1} \cdot\left[1-F_{n}\left(x t_{n}\right)\right] \cdot \exp \left\{(1 / 2) x^{2} t_{n}^{2} h_{n}(x)\right\} \geqq L(x) \quad \text { if } x \in(A, B)
$$

and $n$ is sufficiently large (depending on $x$ ). Furthermore, for all sufficiently large $k, 1-\Phi\left(\beta t_{n_{k}}\right)>\exp \left\{-(1+\gamma / 2) \beta^{2} t_{n_{k}}^{2} / 2\right\}$, by Lemma 1 (ii), and $h_{n_{k}}(\beta)<\gamma / 2$ by (v). Hence

$$
\begin{aligned}
P B_{k} & \geqq L(\beta) \exp \left\{-(1 / 2) \beta^{2} t_{n_{k}}^{2}\left(1+(\gamma / 2)+h_{n_{k}}(\beta)\right)\right\}>L(\beta) \exp \left\{-t_{n_{k}}^{2} / 2\right\} \\
& =L(\beta)\left(\log s_{n_{k}}^{2}\right)^{-1} \sim L(\beta)(2 \log c)^{-1} k^{-1} .
\end{aligned}
$$

Therefore $\sum_{k \geqq K_{1}} P B_{k}=\infty$.

But from the definitions it is clear that $P B_{k} \leqq P A_{k}+P C_{k}$. From the results above, then, it must be the case that $\sum_{k \geqq K_{1}} P A_{k}=\infty$. However, the events $A_{k}$ are independent, so $P\left[A_{k}\right.$ i.o. $]=1$ by the Borel Zero-One Law. That is to say,

$$
\limsup _{k \rightarrow \infty}\left(S_{n_{k}}-S_{n_{k-1}}\right) /\left(u_{k} v_{k}\right)>1-\varepsilon \text { a.e. }
$$

which, in turn, implies

$$
\limsup _{k \rightarrow \infty}\left(S_{n_{k}}-S_{n_{k-1}}\right) /\left(s_{n_{k}} t_{n_{k}}\right)>(1-\varepsilon)\left(c^{2}-1\right)^{1 / 2} \cdot c^{-1} \text { a.e. }
$$

If one notes that $F_{n}(-x)=1-P\left[-S_{n} \leqq x s_{n}\right]$, then it will be evident from the last hypothesis of the theorem that the sequence $\left\{-X_{n}\right\}$ satisfies the conditions of the first part of the theorem: Hence $\lim \sup _{n \rightarrow \infty}-S_{n} /\left(s_{n} t_{n}\right) \leqq 1$ a.e., from which it easily follows that $\lim \inf _{k \rightarrow \infty} S_{n_{k-1}} /\left(s_{n_{k}} t_{n_{k}}\right) \geqq-c^{-1}$ a.e. Adding this inequality to (6) we have, by virtue of (5), $\lim \sup _{k \rightarrow \infty} S_{n_{k}} /\left(s_{n_{k}} t_{n_{k}}\right)>1-\varepsilon^{\prime}$ a.e. (2) follows since $\varepsilon^{\prime}$ is arbitrarily close to zero. Q.E.D.

3. Some consequences of the main theorem. An immediate, but useful, consequence of Theorem 1 is the following:

TheOREM 2. Let $X_{1}, X_{2}, \ldots$ be independent r.v. with $E X_{n}=0$ and $E X_{n}^{2} \equiv \sigma_{n}^{2}<\infty$. Define $S_{n}, s_{n}, t_{n}$ and $F_{n}$ as in Theorem 1. Suppose that $1-F_{n}\left(x t_{n}\right) \sim 1-\Phi\left(x t_{n}\right)$ and $F_{n}\left(-x t_{n}\right) \sim \Phi\left(-x t_{n}\right)$ for all $x$ in some interval containing 1 . Then (2) holds provided $\sigma_{n} / s_{n} \rightarrow 0$ and $s_{n} \rightarrow \infty$ as $n \rightarrow \infty$. 
Remarks. It easily follows from Theorem 2 that if $X_{n}$ is $N\left(0, \sigma_{n}^{2}\right)$ with $s_{n} \rightarrow \infty$ and $\sigma_{n} / s_{n} \rightarrow 0$, then (2) holds. Such a conclusion is similar to, but not as strong as, a result of Hartman [7], who showed that one can replace " $\sigma_{n} / s_{n} \rightarrow 0$ " by " $\lim \sup \sigma_{n} / s_{n}<1$ ".

From Theorem 2 it also follows that if $X_{1}, X_{2}, \ldots$ are independent, identically distributed with mean 0 , variance 1 , and with a moment-generating function that exists in a neighborhood of zero, then $\lim \sup \left(X_{1}+\cdots+X_{n}\right) /(2 n \log \log n)^{1 / 2}=1$ a.e. This statement is not as powerful as the well-known result of Hartman and Wintner [6] which shows that the assumption regarding the moment-generating function is superfluous. However, it might be worthwhile to point out that the Hartman-Wintner result can be derived from Theorem 2 in an indirect way by noting that the law of the iterated logarithm holds for sequences of independent $N(0,1)$ random variables and then applying an invariance principle of Stassen [14].

Under suitable conditions, the law of the iterated logarithm holds for normal random variables, as indicated above. One might wonder if such results are true for sums of independent random variables which are asymptotically normal, or, more precisely, one could ask: Is Lindeberg's condition sufficient to imply the law of the iterated logarithm? This question has been answered in the negative, even for certain sequences of bounded random variables; furthermore, not even a onesided result can be stated in general (see Theorem 1 of [10], or [15]).

Recently, however, some results have appeared which show that the law of the iterated logarithm does hold if convergence in the Central Limit Theorem is sufficiently rapid. Two such results are due to Chung [2] and Petrov [12] (these will be stated precisely later) who use uniform bounds on $\left|F_{n}(x)-\Phi(x)\right|$ to get twosided exponential bounds for $1-F_{n}(x)$, and then use techniques similar to those of Kolmogorov to demonstrate their results. It might be well to mention that Petrov [13] has a similar result for symmetric random variables which may not have finite second moments.

The remainder of this section will be devoted to proving the law of the iterated logarithm results related to the Central Limit Theorem or, more specifically, to bounds of the Berry-Esseen type, using Theorem 2. Toward this end we define, for each $n \geqq 1$,

$$
M_{n}=\sup _{-\infty<x<\infty}\left|F_{n}(x)-\Phi(x)\right| \text { and } g_{n}(\varepsilon)=s_{n}^{-2} \sum_{k=1}^{n} \int_{\left|X_{k}\right| \geq \varepsilon s_{n}} X_{k}^{2},
$$

where $\varepsilon>0$ (this is Lindeberg's function).

For any $a>0$, then,

$$
\begin{aligned}
&\left|\frac{1-F_{n}\left(a t_{n}\right)}{1-\Phi\left(a t_{n}\right)}-1\right| \leqq\left[1-\Phi\left(a t_{n}\right)\right]^{-1} M_{n} \text { and } \\
&\left|F_{n}\left(-a t_{n}\right)\left[\Phi\left(-a t_{n}\right)\right]^{-1}-1\right| \leqq\left[1-\Phi\left(a t_{n}\right)\right]^{-1} M_{n} .
\end{aligned}
$$

THEOREM 3. Let $X_{1}, X_{2}, \ldots$ be independent r.v. with $E X_{n}=0$ and $E X_{n}^{2} \equiv \sigma_{n}^{2}<\infty$. 
Define $S_{n}=X_{1}+\cdots+X_{n}, s_{n}^{2}=E S_{n}^{2}, t_{n}^{2}=2 \log \log \left(s_{n}^{2}\right)$, and define $M_{n}$ as in the preceding paragraph.

If $\sigma_{n} / s_{n} \rightarrow 0, s_{n} \rightarrow \infty$ and $M_{n}=o\left(1-\Phi\left(a t_{n}\right)\right)$ for all a in some interval containing 1 , then (2) holds.

Proof. This result follows immediately from Theorem 2, since it is evident from (7) that the tail-equivalence conditions of that theorem hold.

Some conditions weaker than those of Theorem 3, but perhaps more readily verified in specific cases, are given in Corollary 1.

COROLlaRY 1. Let $\left\{X_{n}\right\}$ be independent r.v. with $E X_{n}=0$ and $E X_{n}^{2} \equiv \sigma_{n}^{2}<\infty$.

Define $S_{n}, s_{n}, t_{n}, F_{n}$ and $M_{n}$ as in the statement of Theorem 3. Then any of the following conditions will ensure that (2) holds:

(i) (Petrov [12]) $\sigma_{n} / s_{n} \rightarrow 0, s_{n} \rightarrow \infty$, and $M_{n}=O\left(\left(\log s_{n}^{2}\right)^{-1-\delta}\right.$ ) for some $\delta>0$.

(ii) $\left(s_{n}^{2} f\left(s_{n}\right)\right)^{-1} \sum_{k=1}^{n} E\left\{X_{k}^{2} \cdot f\left(X_{k}\right)\right\}=o\left(1-\Phi\left(a t_{n}\right)\right)$ as $n \rightarrow \infty$ for all $a$ in some interval containing 1 , where $f \geqq 0$ is an even function such that $\lim _{x \rightarrow \infty} f(x)=\infty$, $x / f(x)$ is defined on $(0, \infty)$, and both $f$ and $x / f(x)$ are nondecreasing on $(0, \infty)$.

(iii) (Chung, p. 218 of [2].) $s_{n}^{-3} \sum_{k=1}^{n} E\left|X_{k}\right|^{3}=o\left(\left(\log s_{n}^{2}\right)^{-1-\delta}\right)$ for some $\delta>0$.

(iv) For some $0<\lambda<\infty, E\left|X_{n}\right|^{3} \leqq \lambda \sigma_{n}^{2}$, for all $n \geqq 1$, and $s_{n} \rightarrow \infty$.

(v) There exists a sequence of positive numbers $\varepsilon_{n}=o\left(1-\Phi\left(a t_{n}\right)\right)$ for all a in some interval containing 1 such that $g\left(\varepsilon_{n}\right) \leqq \varepsilon_{n}^{3}$ for all sufficiently large $n$.

Remark. Conditions (i)-(iv) become progressively easier to verify, but provide progressively weaker results. Each of the conditions (i)-(v), however, implies that Lindeberg's criterion holds. In particular, (v) shows that an iterated logarithm result holds if Lindeberg's function tends to zero in a specific fashion.

Proof. (i) It is well known that $1-\Phi(x) \sim(2 \pi)^{-1 / 2} x^{-1} \exp \left\{-x^{2} / 2\right\}$ as $x \rightarrow \infty$. So, for any $0<a<(1+\delta)^{1 / 2}$,

$$
\left(1-\Phi\left(a t_{n}\right)\right)^{-1}\left(\log s_{n}^{2}\right)^{-1-\delta} \sim(2 \pi)^{1 / 2} a t_{n}\left(\log s_{n}^{2}\right)^{a^{2}-1-\delta} \rightarrow 0
$$

as $n \rightarrow \infty$. Hence (i) is a consequence of Theorem 3 .

(ii) Note that, for $0<\varepsilon<1, f\left(s_{n}\right) / f\left(\varepsilon s_{n}\right)=\varepsilon^{-1} \cdot\left(\varepsilon s_{n} / f\left(\varepsilon s_{n}\right)\right) \cdot\left(s_{n} / f\left(s_{n}\right)\right)^{-1} \leqq \varepsilon^{-1}$. So, clearly, $g_{n}(\varepsilon) \leqq\left(\varepsilon s_{n}^{2} \cdot f\left(s_{n}\right)\right)^{-1} \sum_{k=1}^{n} E\left\{X_{k}^{2} \cdot f\left(X_{k}\right)\right\} \rightarrow 0$ for each $\varepsilon>0$.

Thus Lindeberg's condition is valid, implying $s_{n} \rightarrow \infty$ and $\sigma_{n} / s_{n} \rightarrow 0$. Furthermore, by a result of Petrov [11], there exists an absolute constant $C$ such that $M_{n} \leqq C\left(s_{n}^{2} \cdot f\left(s_{n}\right)\right)^{-1} \sum_{k=1}^{n} E\left\{X_{k}^{2} \cdot f\left(X_{k}\right)\right\}$ if $f$ satisfies the conditions of (ii). The desired result is now immediate from Theorem 3.

(iii) follows directly from (ii), letting $f(x)=|x|$.

(iv) is a consequence of (iii).

(v) Let $\varepsilon>0$. For sufficiently large $n, \varepsilon_{n} \leqq \varepsilon$ so that $g_{n}(\varepsilon) \leqq g_{n}\left(\varepsilon_{n}\right) \leqq \varepsilon_{n}^{3} \rightarrow 0$. Hence Lindeberg's condition holds, and therefore $\sigma_{n} / s_{n} \rightarrow 0$ and $s_{n} \rightarrow \infty$. But a theorem of Berry (p. 136 of [1]) states that an absolute constant $D$ exists such that $M_{n} \leqq D \varepsilon$ if $g_{n}(\varepsilon) \leqq \varepsilon^{3}$. The conditions of Theorem 3 are clearly satisfied. Q.E.D. 
4. On Kolmogorov's theorem. As mentioned in the introduction, a proof of Kolmogorov's Law of the Iterated Logarithm will be given in this section, without the use of Kolmogorov's exponential bounds (see p. 255 of [9]). The following result of Feller [3] will be required: let $\left\{X_{n}\right\}$ be a sequence of independent random variables, each with mean zero and finite variance. Let $S_{n}=X_{1}+\cdots+X_{n}$, $s_{n}^{2}=E S_{n}^{2}, F_{n}(x)=P\left[S_{n} \leqq x \cdot s_{n}\right]$ and suppose positive constants $\lambda_{n}>0$ exist such that $\max _{k \leqq n}\left|X_{k}\right| \leqq \lambda_{n} s_{n}$ and $\lambda_{n} \rightarrow 0$. Then, for any real number $x$ such that $0<\lambda_{n} x<1 / 12$, it is true that

$$
1-F_{n}(x)=\exp \left\{-(1 / 2) x^{2} Q_{n}(x)\right\} \cdot\left\{1-\Phi(x)+\theta \lambda_{n} \exp \left\{-(1 / 2) x^{2}\right\}\right\}
$$

here $|\theta|<9$ and $Q_{n}(x)=\sum_{k \geqq 1} q_{n k} x^{k}$ where the coefficient $q_{n k}$ depends only on the first $k$ moments of $X_{1}, X_{2}, \ldots, X_{n}$-in fact, $\left|q_{n k}\right| \leqq(1 / 7)\left(12 \lambda_{n}\right)^{k}$, so $\left|Q_{n}(x)\right|$ $\leqq(1 / 7)\left(12 \lambda_{n} x\right) /\left(1-12 \lambda_{n} x\right) \rightarrow 0$.

Using this result we will prove

TheOREM 4 (Kolmogorov [8]). Let $\left\{X_{n}\right\}$ denote a sequence of independent r.v. each with mean zero and finite variance. Let $S_{n}=X_{1}+\cdots+X_{n}, s_{n}^{2}=E S_{n}^{2}$, and $t_{n}^{2}=2 \log \log \left(s_{n}^{2}\right)$. Assume $s_{n} \rightarrow \infty$ and $\max _{k \leqq n}\left|X_{k}\right| \leqq K_{n} s_{n} t_{n}^{-1}$ where $0<K_{n} \rightarrow 0$. Then

$$
\limsup _{n \rightarrow \infty} S_{n} /\left(s_{n} t_{n}\right)=1 \text { a.e. }
$$

Proof. Clearly $E X_{n}^{2} / s_{n}^{2} \leqq K_{n}^{2} / t_{n}^{2} \rightarrow 0$. Let $\lambda_{n}=K_{n} t_{n}^{-1}$. For any $a>0, \lambda_{n}\left(a t_{n}\right)$ $=a K_{n}>0$, so that $a \lambda_{n} t_{n}<1 / 12$ for all sufficiently large $n$. By Feller's result,

$$
\frac{1-F_{n}\left(a t_{n}\right)}{1-\Phi\left(a t_{n}\right)} \cdot \exp \left\{(1 / 2) a^{2} t_{n}^{2} Q_{n}\left(a t_{n}\right)\right\}=1+\left(1-\Phi\left(a t_{n}\right)\right)^{-1} \cdot \theta \lambda_{n} \exp \left\{-(1 / 2) a^{2} t_{n}^{2}\right\} .
$$

However $|\theta|<9$ and the last term of the preceding equation is equivalent to $\theta(2 \pi)^{1 / 2} a t_{n} \lambda_{n} \rightarrow 0$ as $n \rightarrow \infty$, so that conditions (i)-(iv) of Theorem 1 are satisfied if one defines $f_{n}(x)=Q_{n}\left(x t_{n}\right)$. Finally since the sequence $\left\{-X_{n}\right\}$ satisfies the conditions of Feller's result, it is evident that the rest of the hypotheses of Theorem 1 hold by using an argument similar to the one above. Q.E.D.

REMARK. Using the result of Feller [3] to prove Kolmogorov's theorem does not simplify or shorten the overall proof. Like Kolmogorov's exponential bounds (see [8], or p. 255 of [9]), Feller's theorem is a deep result with a rather long proof.

\section{BIBLIOGRAPHY}

1. A. C. Berry, The accuracy of the Gaussian approximation to the sum of independent variates, Trans. Amer. Math. Soc. 49 (1941), 122-136. MR 2, 228.

2. K. L. Chung, A course in probability theory, Harcourt, Brace and World, New York, 1968.

3. W. K. Feller, Generalization of a probability limit theorem of Cramér, Trans. Amer. Math. Soc. 54 (1943), 361-372. MR 5, 125.

4. - An introduction to probability theory and its applications. Vol. I, 2nd ed., Wiley, New York, 1962. 
5. W. K. Feller, An introduction to probability theory and its applications. Vol. II, Wiley, New York, 1966. MR 35 \#1048.

6. P. Hartman and A. Wintner, On the law of the iterated logarithm, Amer. J. Math. 63 (1941), 169-176. MR 2, 228.

7. P. Hartman, Normal distributions and the law of the iterated logarithm, Amer. J. Math. 63 (1941), 584-588. MR 3, 2.

8. A. Kolmogorov, Uber das Gesetz des Iterierten Logarithmus, Math. Ann. 101 (1929), 126-135.

9. M. Loève, Probability theory. Foundations. Random sequences, 3rd ed., Van Nostrand, Princeton, N. J., 1963. MR 34 \#3596.

10. J. Marcinkiewicz and A. Zygmund, Remarque sur la loi du logarithme itéré, Fund. Math. 29 (1937), 215-222.

11. V. V. Petrov, $A$ bound for the deviation of the distribution of a sum of independent random variables from the normal law, Dokl. Akad. Nauk SSSR 160 (1965), 1013-1015=Soviet Math. Dokl. 6 (1965), 242-244. MR 31 \#2754.

12. - On a relation between an estimate of the remainder in the central limit theorem and the law of iterated logarithm, Teor. Verojatnost. i Primenen. 11 (1966), 514-518=Theor. Probability Appl. 11 (1966), 454-458. MR 35 \#3720.

13. - On the law of the iterated logarithm without assumptions about the existence of moments, Proc. Nat. Acad. Sci. U.S.A. 59 (1968), 1068-1072. MR 37 \#3636.

14. V. Strassen, An invariance principle for the law of the iterated logarithm, Z. Wahrscheinlichkeitstheorie und Verw. Gebiete 3 (1964), 211-226. MR 30 \#5379.

15. M. Weiss, On the law of the iterated logarithm, J. Math. Mech. 8 (1959), 121-132. MR 21 \#1639.

\section{UNIVERSITY OF SASKaTCHEWAN, Regina, Canada}

